\title{
Computational Simulation for Decision of Scheduling Period in Reactive Scheduling*
}

\author{
Tatsuhiko SAKAGUCHI ${ }^{* *}$, Toshihide KAMIMURA ${ }^{* * *}$ and Keiichi SHIRASE ${ }^{* * *}$ \\ ${ }^{* *}$ Organization of Advanced Science and Technology, Kobe University, \\ 1-1, Rokkodai, Nada-ku, Kobe 657-8501, Japan \\ E-mail: sakaguchi@mech.kobe-u.ac.jp \\ ${ }^{* *}$ Graduate School of Engineering, Kobe University, \\ 1-1, Rokkodai, Nada-ku, Kobe 657-8501, Japan
}

\begin{abstract}
Unexpected disruptions often occur in the manufacturing systems. The manufacturing systems cannot execute the manufacturing operations in accordance with the predetermined production schedule due to such disruptions. Therefore, a systematic scheduling method is required to cope with such disruptions. In this research, distribution of processing time is described with the normal distribution. The reactive scheduling method for distribution of processing time is proposed in order to modify the predetermined production schedule. And the suitable re-scheduling period is considered through the computational experiments.
\end{abstract}

Key words: Reactive Scheduling, Distribution of Processing Time, Normal Distribution, Tardiness, Flow Time

\section{Introduction}

A production schedule is predetermined before executing manufacturing processes in the manufacturing systems. However, it often happens that the manufacturing system cannot execute in accordance with the predetermined schedule because of the distribution of the processing time. In such case, the initial production schedule has to be modified for satisfying the constraints such as the due date. A dynamic scheduling method is therefore required to cope with such unexpected disruptions. The reactive scheduling method is one of the dynamic scheduling methods that satisfy this requirement ${ }^{(1)}$. A reactive scheduling method based on Genetic Algorithm (GA) was proposed, in the previous research, to modify the initial production schedule when the manufacturing processes delay in the manufacturing systems ${ }^{(2)}$. This method can modify the initial production schedule without aborting the manufacturing processes when operation delays occur. However, in the previous research, the operation delays are applied to only a few operations, and detected before the delayed operations start in the manufacturing systems. In the actual manufacturing system, all operation has possibility of delay. Moreover, it is difficult to detect the operation delay before the manufacturing operations start.

In this research, it is assumed that all operation varies based on the normal distribution and the operation delay is detected just after the operation finishes. The reactive scheduling method is extended in order to deal with such situation. Moreover, by computational simulation, the most suitable scheduling period is analyzed experimentally. 


\section{Normal Distribution Based Processing Time}

\subsection{Description of production scheduling problem}

The following terms are used in the production scheduling problem in this research.

1) Job is the element to be manufactured in the manufacturing system, such as a part, an assembly and a lot. Jobs are represented by $J_{i}(i=1,2, \ldots, m)$.

2) Resource is the element that carries out the manufacturing processes of the jobs. A machine tool, a manufacturing cell and a manufacturing shop are typical examples of the resources. Resources are represented by $R_{j}(j=1,2, \ldots, n)$.

3) Operation represents the manufacturing activity executed by a combination of a job and a resource. A milling process of a part is an example of the operation. Operations are represented by $O_{i, j}^{k, l}$. Operation $O_{i, j}^{k, l}$ is the $k$-th process of job $J_{i}$, and is manufactured $l$-th by resource $R_{j}$.

The scheduling problem is to select the suitable loading sequences of the jobs to the resources based on the objective function. A job-shop type manufacturing system is the subject of this research. In job-shop scheduling problem, there are $n$ different resources and $m$ different jobs to be scheduled. Each job has a predetermined manufacturing sequence. Each resource can deal with only one job at the same time. Typical objective functions in the scheduling problem are to minimize the make-span of the jobs, to minimize the waiting time of the jobs and to minimize the tardiness of the jobs.

\subsection{Definition of Processing Time}

In this research, it is assumed that the processing time in the manufacturing system can be described with the normal distribution. Therefore, the finishing time of the current executed operation vary as shown in Fig. 1. The finishing time is calculated by the following equation.

$$
F T=S T+P T \times N R N_{(\mu, \sigma)}
$$
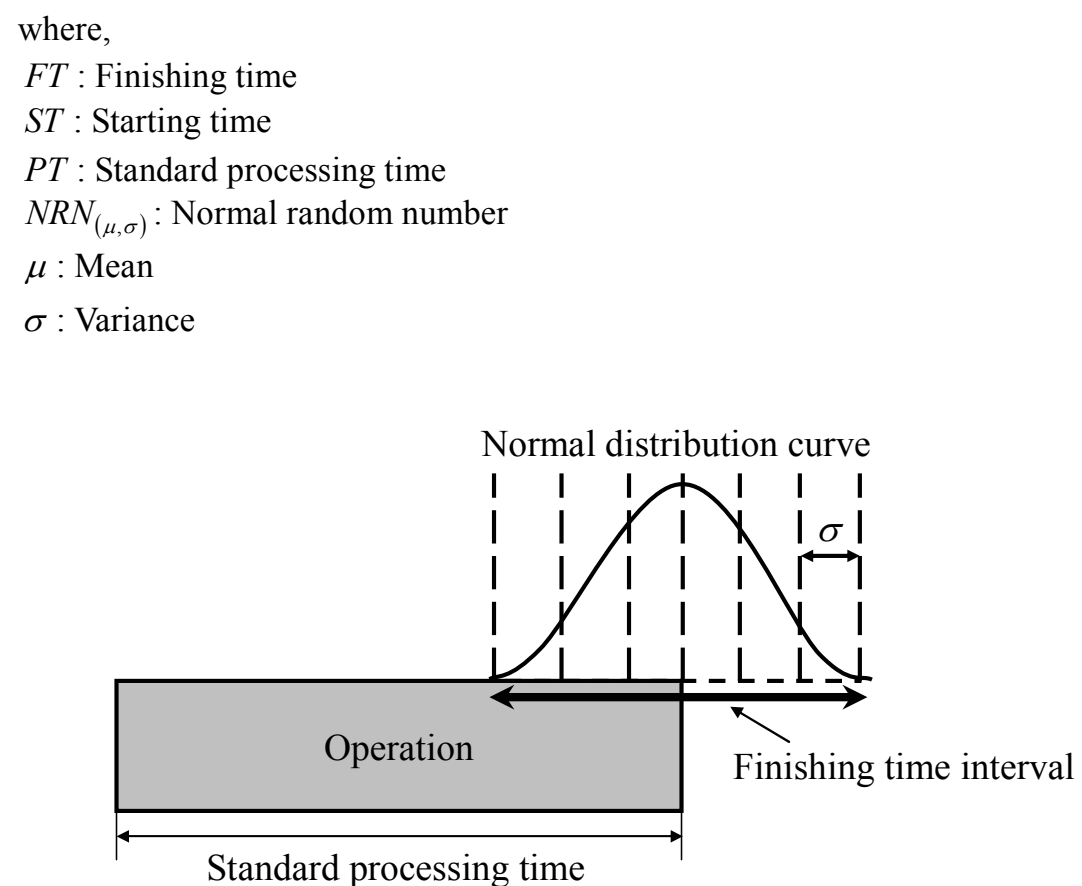

Fig. 1 Processing time based on normal distribution 


\section{GA Based Reactive Scheduling}

\subsection{Estimation of processing time}

In the previous research, a reactive scheduling method modifies the pre-determined initial schedules when operation delay occurs. However, it was assumed that the delay is detected before the delayed operations start in the manufacturing systems. The scheduling system needs to be informed when the operation delay occurs and how long the delay is, before the operations start. In the actual manufacturing processes, it is difficult to predict the length of the delay. The progress of manufacturing operations has to be monitored in order to modify the initial schedule in the context of the actual situation on the manufacturing systems. Moreover, in order to modify the initial schedule when delays occur, the estimated processing time has to be determined.

In this research, the reactive scheduling system detects the operation delays and shortens, and determines the estimated processing time by the following steps. Figure 2 shows the process of these steps.

\section{Step 1: Receive signal}

The reactive scheduling system receives the signal of the starting and finishing time which is sent from the components of the manufacturing system.

\section{Step 2: Delay and shorten detection}

Three cases are considered in the detection of delay of manufacturing processes. They are,

1) The manufacturing operations are executed on schedule in the manufacturing system.

2) The manufacturing operations are finished before the standard processing time. In this case, the processing time is fixed by Step 5 .

3) Delay occurs in the manufacturing system.

For the case 1) and 2), the reactive scheduling process is not executed. On the other hand, for the case 3), the reactive scheduling process is activated in order to modify the initial production schedule.

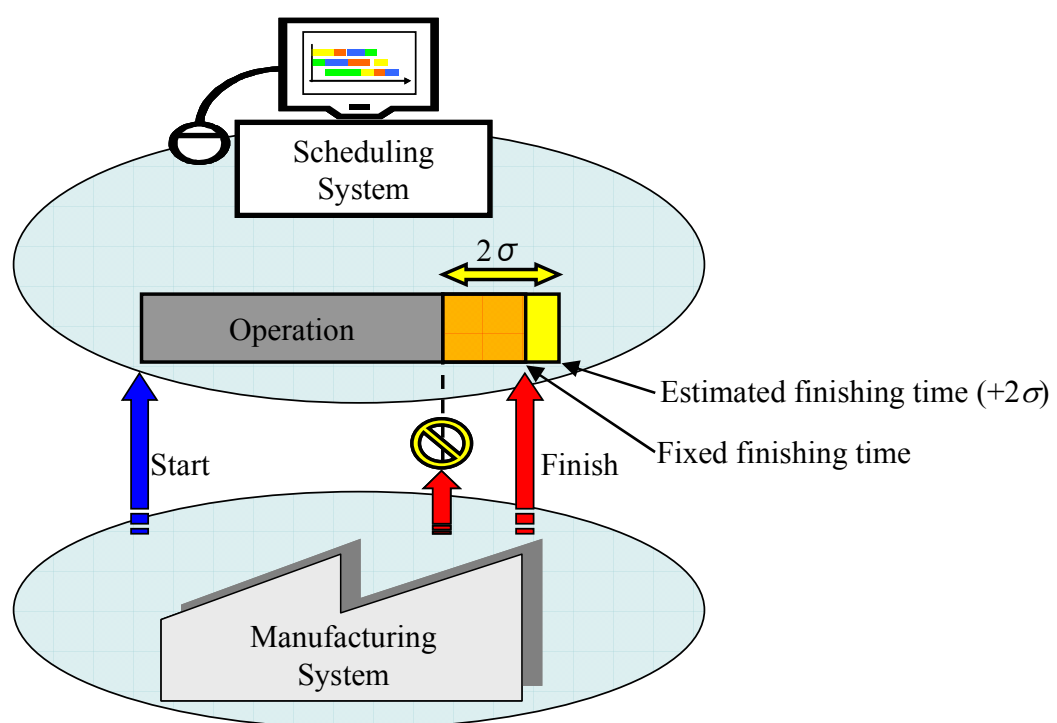

Fig. 2 Estimation of processing time 
Step 3: Estimation of the processing time

If the reactive scheduling system detects the delay of manufacturing processes, the reactive scheduling system sets the estimated processing time in order to modify the initial production schedule, because it is not known when the delayed operation will finish. The estimated processing time of operation is calculated by using the following equation.

$$
E P T=T P+2 \sigma
$$

Where,

$E P T$ : Estimated processing time

$T P$ : Initial processing time on the schedule

By the normal distribution, the probability of the random variable exists in $2 \sigma$ is $95.44 \%$. Therefore, in this research, the estimated processing time is calculated based on $2 \sigma$.

Step 4: Executing reactive scheduling

The reactive scheduling process is repeated until the modified production schedule satisfies the constraint on due date or all the manufacturing operations finish.

Step 5: Fix the processing time

If the finishing signal is received from the manufacturing system, the processing time is fixed and applied the current schedule.

On the other hand, the finishing signal is not received within the $(T P+2 \sigma)$, the estimated processing time is updated the following equation, and the reactive scheduling process is iterated from Step 4.

$$
E P T=T P+3 \sigma
$$

Moreover, if the finishing signal is not received within the $(T P+3 \sigma)$, the estimated processing time is updated the following equation.

$$
E P T=T P+3 \sigma+d t
$$

where $d t$ is the calculation time for one reactive scheduling cycle. It is determined based on the calculation time for one generation of the genetic algorithm in the creation of the initial schedule.

\subsection{Reactive Scheduling Process}

When the operation delays are detected and the production schedule cannot satisfy the constraint of due date, the reactive scheduling process is executed.

The reactive scheduling processes consist of the following five steps.

Step 1: Setting up of present time $T_{i}$

The present time $T_{i}(i=1,2, \ldots)$ is set up.

\section{Step 2: Estimation of computation time $d t$}

The computation time $d t$ is the time in which GA creates a new generation of the populations representing the modified production schedules. The time $d t$ is estimated based on the time needed to generate a new population in the GA based current production scheduling process, and it is modified based on the time for creating the modified 
production schedules through Step 3 to Step 5.

Step 3: Creation of initial population

Two cases are considered in the creation of the initial population constituted of the individuals which represent the production schedules. They are,

1) First activation of the reactive scheduling process at time $T_{1}$.

2) Second or later activations of the reactive scheduling process at time $T_{2}$ or later.

For the cases of 1) and 2), the reactive scheduling process creates the initial population through the Step 3-1 and Step 3-2, respectively.

Step 3-1: First activation of the reactive scheduling process

In the case 1), the reactive scheduling system has only the initial production schedule. Therefore, the reactive scheduling process generates the initial population based on the initial production schedule as shown in Fig. 3 .

Step 3-2: Second or later activations of the reactive scheduling process

In the case 2), the reactive scheduling process can inherit the population created in the previous reactive scheduling process. In other words, the last population of the previous reactive scheduling process can be the initial population. Two cases are considered for the inheritance process of the population as shown in the followings.

Case-A: No operations start between $T_{i}$ and $\left(T_{i}+d t\right)$

If no operations start between $T_{i}$ and $\left(T_{i}+d t\right)$, all the individuals of the last population of the previous reactive scheduling process are inherited to a new reactive scheduling process between $T_{i}$ and $\left(T_{i}+d t\right)$.

Case-B: Some operations start between $T_{i}$ and $\left(T_{i}+d t\right)$

If some operations start between $T_{i}$ and $\left(T_{i}+d t\right)$, the production schedules of these operations should be fixed. Therefore, a new reactive scheduling process can inherit only the individuals, which are consistent with the schedules of the fixed operations, from the last population created in the previous reactive scheduling process. The other individuals are deleted, and new individuals are randomly created from the inherited ones.

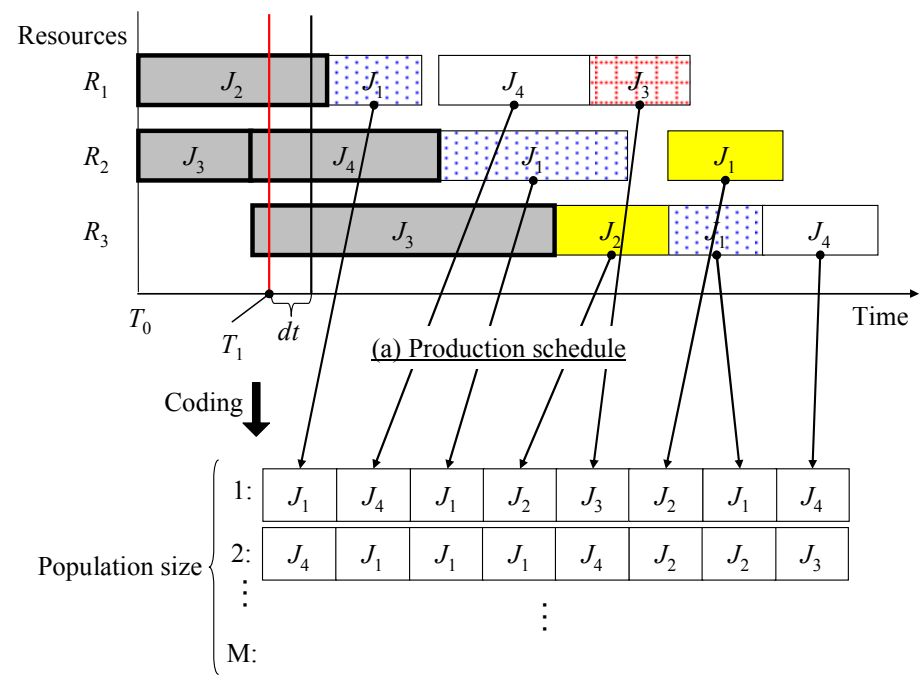

(b) Initial population

Fig. 3 Generation of initial population 
Step 4: Application of genetic operators to the population

The fitness value of each individual is evaluated. The objective function value of the production schedule, which has to be minimized, is selected as the fitness value. Based on the fitness value, the genetic operators, such as selection, crossover and mutation, are applied to the individuals of the population created in Step 3, in order to create new individuals of the next population.

Step 5: Evaluation of modified production schedule

If the minimum objective function value of all the new individuals created in Step 4 is better than the objective function value of the current production schedule, the new modified production schedule is applied for the current production schedule. If the objective function value of the new production schedule satisfies the given criterion, the reactive scheduling process will be terminated.

All the steps from Step 1 to Step 5 are repeated, until the created production schedule satisfies the given criterion or all the manufacturing operations have started in the manufacturing system.

\section{Computational Experiments}

\subsection{Reactive Scheduling System}

A prototype of reactive scheduling system has been implemented and applied to the computational experiments for the job-shop type scheduling problems. Numbers of jobs, manufacturing equipment and operations considered in the experiments were 20, 10 and 200 , respectively. The processing time of each operation in the initial production schedule is set based on the bench-mark problem proposed by Storer, Wu and Vaccari ${ }^{(3)}$. Parameters of GA, such as population size, crossover rate and mutation rate, were 50, 0.5 and 0.1 , respectively. These parameter values were estimated based on preliminary case studies of the job-shop type production scheduling problems.

Parameters of normal distribution, such as $\mu$ and $\sigma$ were 0 and $10 \%$ of standard processing time respectively.

\subsection{Evaluation of Production schedule}

In this research, the objective functions for scheduling are total tardiness and total flow time. In order to consider both the objective functions, the weighted sum of these functions is calculated by the following equation.

$$
O F=\alpha \frac{T T_{i}-T T_{\min }}{T T_{\max }-T T_{\min }}+(1-\alpha) \frac{T F T_{i}-T F T_{\min }}{T F T_{\max }-T F T_{\min }}
$$

where,

$O F$ : Objective function value

$T T_{\max }: \quad$ Maximum value of total tardiness in the population

$T T_{\min }: \quad$ Minimum value of total tardiness in the population

$T T_{i}$ : $\quad$ Total tardiness of $i$-th schedule

$T F T_{\max }:$ Maximum value of total flow time in the population

$T F T_{\min }: \quad$ Maximum value of total flow time in the population

$T F T_{i}$ : $\quad$ Total flow time of $i$-th schedule

$\alpha: \quad$ Weight $(0<\alpha<1)$

The objective of the scheduling is to minimize total tardiness and total flow time. 
Therefore, the objective function value $O F$ has to be minimized.

\subsection{Schedule Conditions}

In this research, the manufacturing system is considered as a job-shop type system. Ten products are manufactured by the manufacturing system in morning and afternoon respectively. Identical products are manufactured. Figure 4 shows the Gantt chart of the initial schedule. The products are shipped twice a day, in the morning and in the afternoon. The products which are scheduled to be manufactured in afternoon can be processed ahead of schedule. The main objective of scheduling is to satisfy the due date. And at the same time, it is expected to minimize the flow time.

The initial schedule was created by the genetic algorithm. The schedule for the morning was created by optimizing the total flow time. The schedule for the afternoon was set with the same sequence of job in each resource as the morning schedule.

The due date of the morning products and the afternoon products were set as 1800 and 3600 respectively. The initial schedule can satisfy the due date.

The manufacturing operations were simulated as 60 times the real-time.

\subsection{Results and Discussion}

Computational experiments of the reactive scheduling have been carried out five times within the same experimental conditions, because GA based scheduling method is a probabilistic search technique and has random computational operations in creating population. Weight $\alpha$ for calculating objective function value was set $0,0.8$ and 1. Figure 5, Fig. 6 and Fig. 7 are the results of the computational experiment for each $\alpha$ value. $\mathrm{X}$ axis and $\mathrm{Y}$ axis of each figure shows time and objective function values respectively. The tardiness and the flow time were increased due to the operational delay within several seconds after the manufacturing operations were executed in manufacturing system. The reactive scheduling system was activated to improve the production schedule. Throughout the simulation, those two objective functions would be fluctuating due to the delay of manufacturing operations and improvement of schedule.

The tardiness and the flow time were most shortened when the weight $\alpha$ is set as 0 . Table 1 shows the total tardiness and the total flow time when the reactive scheduling process finished.

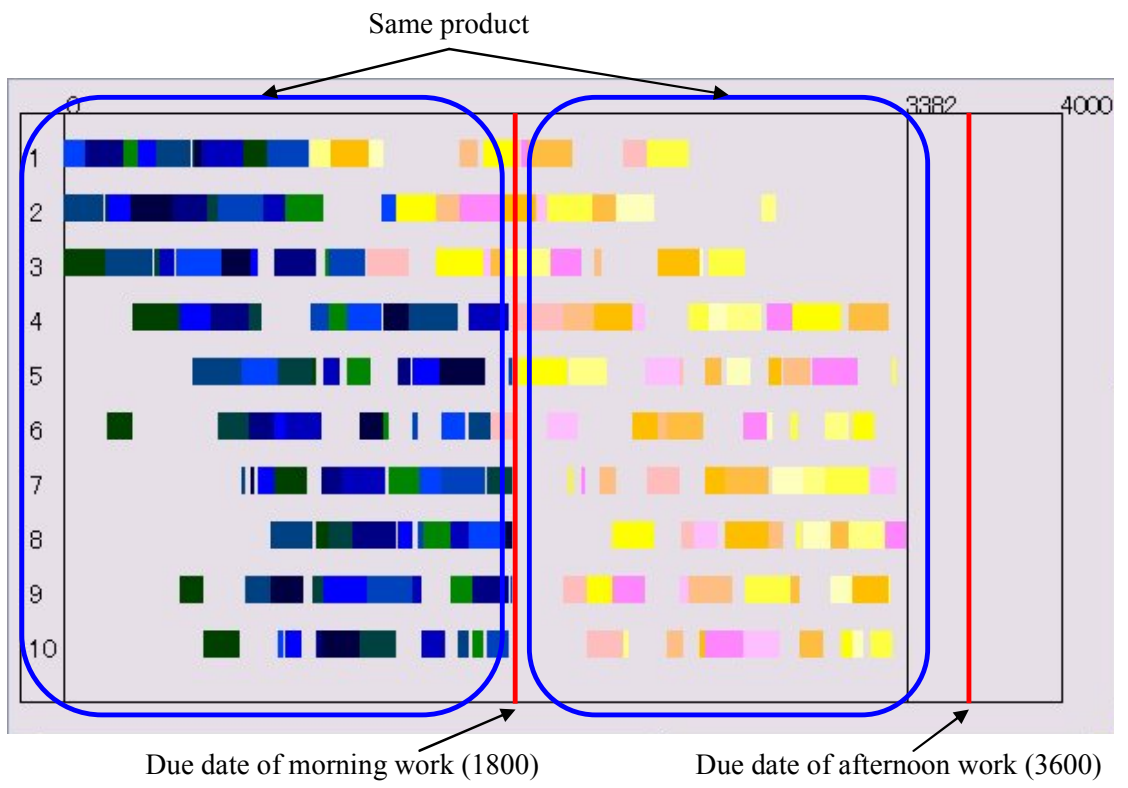

Fig. 4 Gantt chart of initial schedule 


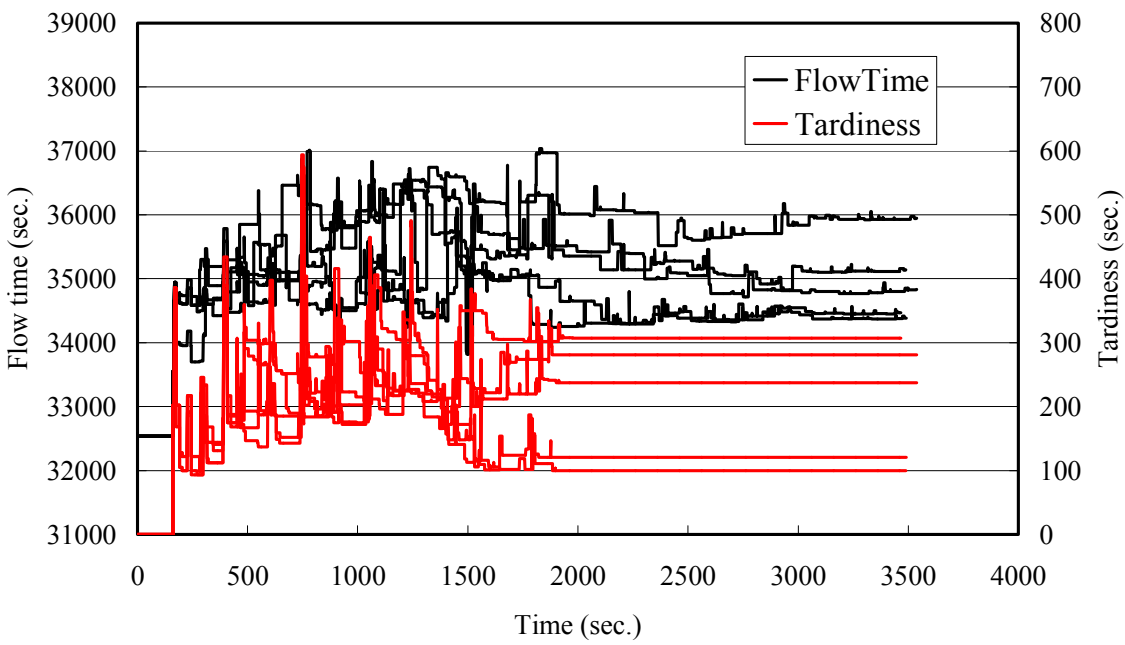

Fig. 5 Results of experiments (Tardiness: 1, Flow time: 0)

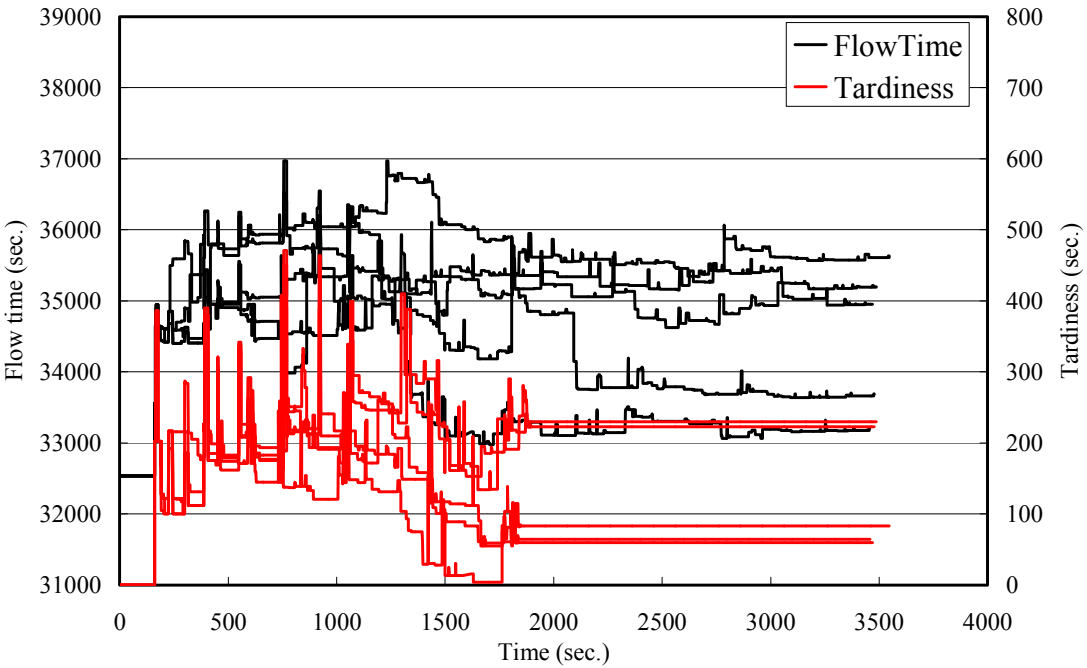

Fig. 6 Results of experiments (Tardiness: 0, Flow time: 1)

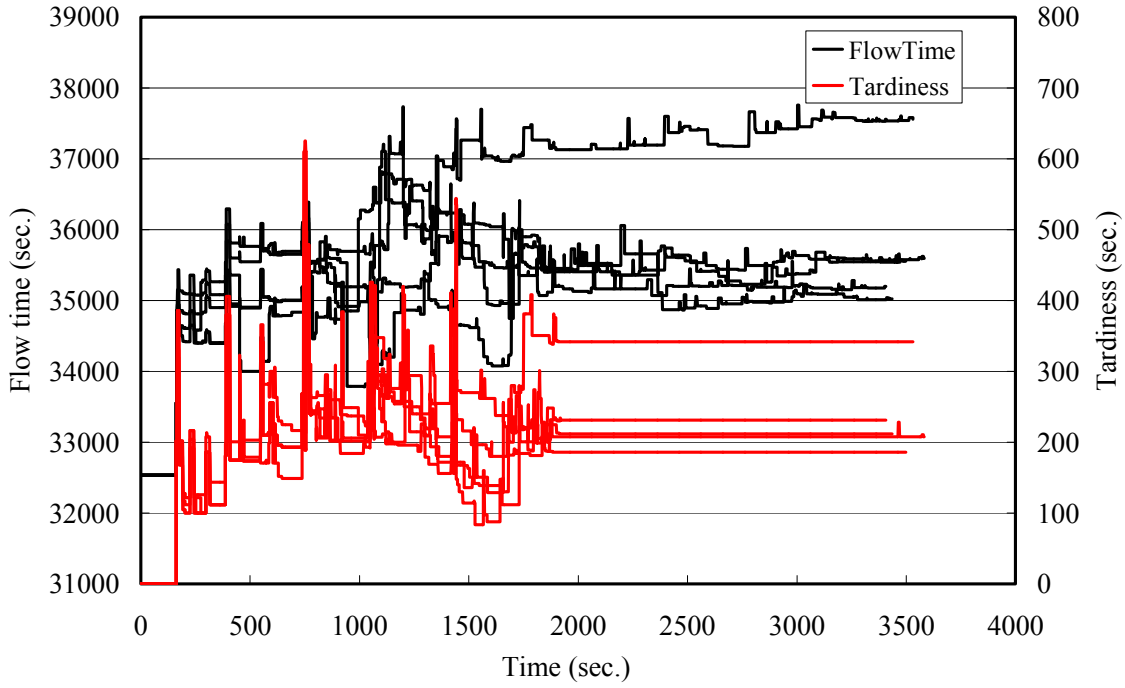

Fig. 7 Results of experiments (Tardiness: 0.8, Flow time: 0.2 ) 
Table 1 Results after reactive scheduling

\begin{tabular}{|c|c|c|}
\hline$\sigma$ settings & Total tardiness (sec.) & Total flow time (sec.) \\
\hline $\begin{array}{c}\text { Tardiness: } 1, \\
\text { Flow time: } 0\end{array}$ & 209.2 & 34955.0 \\
\hline $\begin{array}{c}\text { Tardiness: } 0, \\
\text { Flow time: } 1\end{array}$ & $\mathbf{1 3 2 . 0}$ & $\mathbf{3 4 5 3 3 . 2}$ \\
\hline $\begin{array}{c}\text { Tardiness: } 0.8, \\
\text { Flow time: } 0.2\end{array}$ & 235.8 & 35787.8 \\
\hline
\end{tabular}

In every case, the total tardiness and the total flow time were not being modified in the afternoon that much. It is considered that the morning products could not satisfy their due date because the schedules of the afternoon products were allocated ahead of them. This is the reason that the total tardiness and the total flow time were larger than the distribution of processing time in early time of reactive scheduling. This situation was confirmed through the Gantt chart.

In the future research, the constraints for inhibiting the schedules of the afternoon products being forwarded should be considered. Or the range of schedule which is modified should be refined. For example, the reactive scheduling process in early morning aims only for the morning products.

\section{Conclusion}

This research deals with the distribution of processing time in manufacturing system. The distribution of processing time is evaluated based on the normal distribution. The reactive scheduling system modifies the initial schedule just after the operation delay occurred, by monitoring the progress of the manufacturing operations. The scheduling objective is to minimize both the total tardiness and the total flow time. The prototype of reactive scheduling system was implemented, and some computational experiments were carried out. By analyzing the results of the experiments, the suitable scheduling period is verified for job-shop type manufacturing system.

\section{Acknowledgement}

A part of this work was supported by the Grant-in-Aid for Scientific Research (B) No. 17360063 of the Japan Society for the Promotion of Science.

\section{References}

(1) Smith, S. F., OPIS: A methodology and architecture for reactive scheduling, intelligent scheduling, (1994), pp. 29-66, Morgan kaufmann publishers.

(2) Tanimizu, Y., Sugimura, N., A study on reactive scheduling based on genetic algorithm, Proceedings of the 35th CIRP International Seminar on Manufacturing Systems, (2002), pp. 219-224.

(3) Storer, R. H., Wu, D. D., Vaccari, R., New search spaces for sequencing instances with application to job shop scheduling, Management science 38, (1992), pp. 1495-1509. 